\title{
Current epidemiology of KSHV infection in American men who have sex with men
}

\author{
Nazzarena Labo ${ }^{1,2^{*}}$, Wendell Miley ${ }^{1}$, Maya Kesler $^{2}$, Lisa Jacobson $^{2}$, Denise Whitby ${ }^{1}$ \\ From $12^{\text {th }}$ International Conference on Malignancies in AIDS and Other Acquired Immunodeficiencies \\ (ICMAOI) \\ Bethesda, MD, USA. 26-27 April, 2010
}

\section{Background}

The introduction of highly active antiretroviral therapy (HAART) has resulted in a dramatic decline in the incidence of AIDS-associated Kaposi sarcoma (KS) among men who have sex with men (MSM). The decline had been associated with increased CD4+ cell counts; however, the effect of HAART on the epidemiology of the Kaposi sarcoma-associated herpesvirus (KSHV) has not been investigated. We describe here the prevalence of KSHV in HIV infected and uninfected MSM across time periods of different prevailing HAART usage.

\section{Subjects, materials, and methods}

The serostatus of 4159 participants in the prospective Multicenter AIDS Cohort Study (MACS) was ascertained using two ELISAs, detecting antibodies against the latently associated nuclear antigen (LANA) and the lytic antigen encoded by ORFk8.1, respectively. KSHV seropositivity was defined as positivity in either assay. The calendar period between 1984 and 1990 was defined as untreated era, while the periods 1990-1995 and 1995-2001 were defined as pre-HAART era and HAART era, respectively. Because the last wave of enrollment in the MACS that occurred in 2001 involved a distinct change in study demographics, a fourth period, 2001-2009 was defined as current era.

\section{Results}

The study population was composed of $2920 \mathrm{HIV}$-positive and 1239 HIV-negative subjects. The prevalence of KSHV was $54.6 \%$ overall (95\% CI: $53.0-56.1 \%$ ), $67.8 \%$ (95\% CI: 66.0-69.5\%), in HIV+ and 23.5\% (95\% CI: 21.1$25.9 \%)$ in HIV- subjects. When examining the cohort by

\footnotetext{
*Correspondence: labon@mail.nih.gov

${ }^{1}$ AIDS and Cancer Virus Program, Viral Oncology Section, SAIC-Frederick, $\mathrm{NCl}$-Frederick, Frederick, MD, USA

Full list of author information is available at the end of the article
}

calendar time, the prevalence was 52.9\% (95\% CI: 51.2$54.6 \%$ ) in the untreated era, $67.5 \%$ (95\% CI: 59.3-75.1\%) in the pre-HAART era, $52.7 \%$ (95\% CI: 35.4-69.9\%) in the HAART era, and 59.7\% (56.0-63.4\%) in the current era.

\section{Conclusions}

The overall prevalence of KSHV in MSM enrolled in the MACS did not vary significantly from 1984 through 2009, indicating that the decline in incidence of KS seen over the same calendar periods in this population is not associated with a decline in KSHV prevalence. The observation is significant because of the potential excess morbidity risk associated with KSHV in an aging population, particularly among HIV/ KSHV co-infected individuals, notwithstanding effective anti-HIV treatment. To elucidate the mechanisms sustaining the high prevalence of KSHV amongst MSM, KHSV incidence in time and risk factors associated with KSHV infection are being investigated.

\section{Acknowledgements \\ This article has been published as part of Infectious Agents and Cancer Volume 5 Supplement 1, 2010: Proceedings of the $12^{\text {th }}$ International Conference on Malignancies in AIDS and Other Acquired \\ Immunodeficiencies (ICMAOI). The full contents of the supplement are available online at http://www.biomedcentral.com/1750-9378/5?issue=S1.}

\section{Author details}

${ }^{1}$ AIDS and Cancer Virus Program, Viral Oncology Section, SAIC-Frederick, NCI-Frederick, Frederick, MD, USA. ${ }^{2}$ Department of Epidemiology, Johns Hopkins School of Public Health, Baltimore, MD, USA.

Published: 11 October 2010

\section{doi:10.1186/1750-9378-5-S1-A13}

Cite this article as: Labo et al:: Current epidemiology of KSHV infection in American men who have sex with men. Infectious Agents and Cancer 2010 5(Suppl 1):A13. 